\title{
Nose abscess after thread rhinoplasty: a case report
}

\author{
Al Farabi Lee Jaafar, MD, FPSCS, FPCS, FPSGS \\ Department of Cosmetic Surgery, Philippine Society for Cosmetic Surgery, Centuria Medical Makati, Makati City, Metro Manila, Philippines
}

\begin{abstract}
A 45-year old female patient developed progressive nose swelling and tenderness after undergoing non-surgical rhinoplasty using polydioxanone threads. Surgical drainage procedure of the nose was done. Intra-operative findings revealed abscess formation over the nasal dorsum extending inferiorly to the nasal tip, supra-septal region and columella. Pus and necrotic materials were evacuated. Culture and sensitivity revealed Propionibacterium acnes sensitive to Ciprofloxacin. Repair of the tip was done with re-approximation of the lower lateral cartilages. The patient recovered with unremarkable post-operative course. Thread use is fast becoming a trend as an option to non-surgical filler and surgical rhinoplasty. Although threads are generally safer than fillers and the technique for insertion is simpler than implant based procedures, surgical aseptic and antiseptic methods should still be strictly applied to prevent the complication of infection.
\end{abstract}

Keywords: adverse effects; dermal fillers; polydioxanone; surgical wound infection

\section{Introduction}

From the first description of Asian augmentation rhinoplasty by Singaporean surgeon Dr. Khoo [1] in 1964, this procedure has since grown to become one of the most common cosmetic surgeries performed in Asia. With the nose being on the center of the face, it is typically the first facial feature noticed or seen. Its size and shape ultimately dictate the balance of the face in relation to other facial features.

As a vast continent comprising a variety of races, Asian noses vary in height/size and shape. The spectrum ranges from the "high-narrow" Caucasian-like noses of Western Asia and Indian subcontinents, to the relatively "low-narrow" and "low-wide" noses of the East Asian and South-East Asian counterparts respectively. These nasal traits among Asians have divided the continent into "reduction rhinoplasty" for West Asia and "augmentation rhinoplasty" for the Eastern part of Asia. Increase in the demand for augmentation rhinoplasty in East Asia was brought about by the popularity of western influence in the media, personal reasons, and socio-economic competitiveness in the job [2].

Asian augmentation rhinoplasty techniques include surgical rhinoplasty (with or without implants) and the less invasive type using injectable gel fillers and threads. Filler nose reshaping is getting the attention, as more and more patients are being enticed to the promise of "fast office procedure" and "shortdowntime".

While fillers are generally safe and easy to administer, complications can happen [3]. Various fillers have different side effect profiles. The greatly feared complication is the irreversible blindness caused by vascular occlusion from accidental intravascular injection [4]. A review published by the American Society of Dermatological Surgery in 2015 revealed that more than $25.5 \%$ of the reported cases of blindness caused by fillers were done in the nasal area, second only to the glabellar region $(38.8 \%)$ [5]. This rare yet serious pitfall of fillers, has led to the 
rise of another non-surgical alternative, the "threads rhinoplasty".

Threads made of polydioxanone (PDO) or PDO, are commonly used in facial enhancements like face, brows, and nose to produce the effect of lifting or shaping. This material is a synthetic polymer absorbable monofilament suture that has an absorption period of 120 to 180 days. A popular technique emerging in Asia is the "Hiko" (high nose in Korean) nose lift. Multiple absorbable PDO threads on blunt cannulas are inserted into a tiny needle pricked hole on the skin of the nose. This technique augments the nasal bridge or dorsum, as well as increase the projection of the tip. The threads are placed in the supraperiosteal/chondrial plane, exactly where fillers or implants are placed [6].

Threads when inserted in multiple numbers on the nose mimic the effect of an implant. In non-surgical face lifting, they also mechanically lift the surrounding tissues as in the case of threads with cogs and barbs, or stimulate dermal collagen for mono-threads. Even if the absorption is less than 6 months, the resulting fibrosis or collagen formation increases the duration of its effect (some claiming to last up to 2 years)[7]. Unlike fillers, threads have no propensity to migrate, thus giving it a better lasting aesthetic outcome. Fillers eventually results to widening of the bridge (lateral migration) and overhanging of the columella (inferior migration). This migration gives the "avatar" and "witch-look" appearance common among patients with nose fillers weeks after treatment. For threads, there is no over-correction, as it does not move or expand in tissues. Very importantly, it does not carry the risk of the dreaded visual complication.

Good outcome of thread procedures is operator dependent. Doctors use various insertion techniques to create different filling, pulling or lifting effects. One of the techniques includes the correct insertion plane to avoid skin bumps (when inserted superficially) or vascular and nerve injuries (when inserted too deeply). Because it is a minimally invasive procedure, operators tend to neglect a simple but important initial step, which is sterility. Since microbes colonize the skin, poor prepping can lead to bacterial inoculation into the subcutaneous tissues underneath. Considering that the nose is within the "dangerous triangle of the face", it is critical to implement strict aseptic and antiseptic processes in order to avoid infections.

This paper presents a case of a 45-year old female who underwent nose-lifting procedure using threads and subsequently developed abscess formation. The complication was misdiagnosed by the aesthetic doctor and was regarded only as normal post-procedure swelling.

\section{Case report}

Six months prior to the current condition, the patient consulted an aesthetic doctor for her low nasal dorsum, and wide/ bulbous tip. A minimally invasive thread rhinoplasty was done. Three days after the procedure, she was off to her normal activities. Two weeks after the thread rhinoplasty, she noted progressive swelling over the nasal bridge. Her doctor gave assurance that it will resolve after one month. The swelling progressed and later was accompanied by pain and tenderness. These resulted to the widening of the nasal dorsum and tip, and bulging of the columella. There were no other symptoms like fever, chills, or headaches. Despite repeated reassurance from the doctor, the patient decided to visit our Centre for second opinion.

Upon initial consultation ( 2 months prior) in our centre, the nose was noted to be disproportionately large in size (Fig. 1). The nasal dorsum and tip were widened. There was bulging of the columella extending supero-posteriorly into the septal region. Immediate surgery to drain the abscess was advised but the patient opted for a conservative management. She was prescribed oral anti-biotic (Co-amoxiclav $625 \mathrm{mg} 1$ tablet every 12 hours for 7 days) and was lost to follow-up.

During her second consultation (1 day before surgery), the patient was conscious, coherent, and afebrile. Physical examination was centered on the face. The nasal dorsum was widened to $2.6 \mathrm{~cm}$ with an anterior projection height of $3.0 \mathrm{~cm}$ at the junction of the upper third of the nose. The lateral borders of the nasal dorsum (bridge) were barely $0.3 \mathrm{~cm}$ from the medial canthus of both eyes. Intercanthal distance was $3.2 \mathrm{~cm}$. The lower third of the nose was also widened and antero-inferiorly projected creating a "witch-look" appearance (Fig. 2). The nasal tip was bulbous and had an inter-alar width of $5.0 \mathrm{~cm}$. The columella was bulging inferiorly and widened to $1.5 \mathrm{~cm}$. Internal examination revealed bilateral bulging and thickening of the septum. There was no hyperemia or redness of the nose noted despite the massive swelling but there was persistent tenderness on pressure.

The patient underwent "open rhinoplasty" under local anesthesia for drainage of the nasal abscess. Two days prior, she was loaded with oral ciprofloxacin $500 \mathrm{mg}$ for 2 doses. Upon opening, an estimated $6.0 \mathrm{ml}$ of bloody-purulent material was evacuated from the supra-periosteal region of the nasal dorsum, the supra-perichondrial region of the tip, and the septum. The surrounding tissues were edematous with bleeders oozing. 

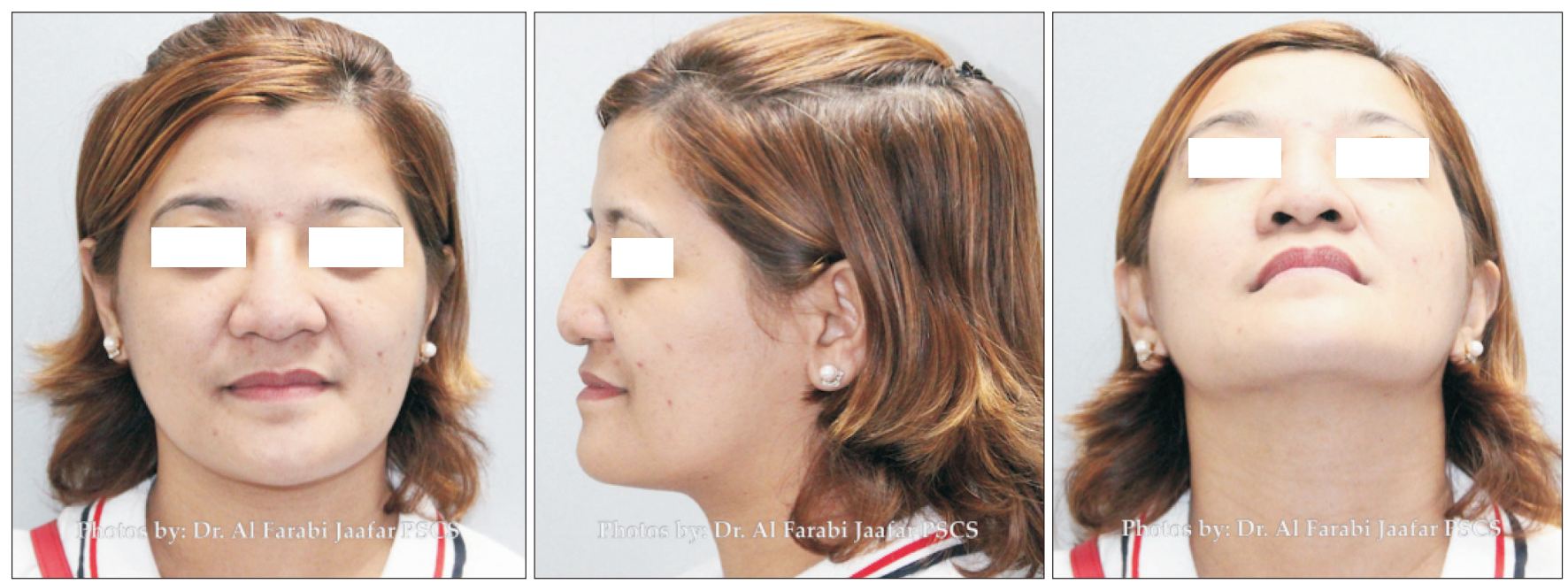

Fig. 1. Initial consultation (2 months before surgery).
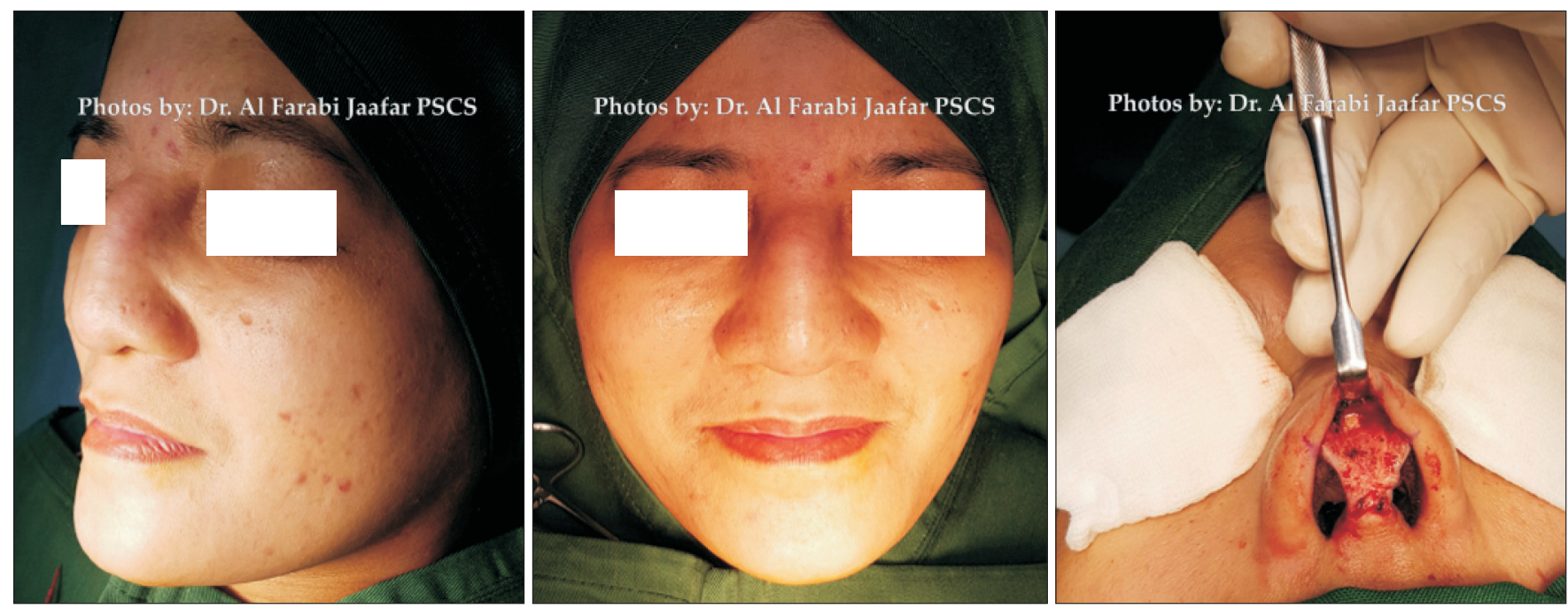

Fig. 2. During surgery photos. Thread lift complications.

Curettage was done to remove necrotic tissues. There were no more threads found. Inter-domal sutures were applied using nylon 5-0 to prevent collapse of the tip. The bilateral rim and columellar incisions were closed with loose stitches using nylon 5-0 simple interrupted. The Procedure was tolerated well (Fig. $3)$.

Patient was discharged an hour after surgery and was managed with oral ciprofloxacin $500 \mathrm{mg} /$ tablet, 1 tablet every 12 hours for 13 more days and celecoxib $400 \mathrm{mg} /$ capsule, 1 capsule once a day for pain relief. Post-operative course was unremarkable with minimal swelling noted on the 3rd post-operative day. Culture and sensitivity examination of the pus material sent to the laboratory showed high growth of gram-positive Propionibacterium acnes sensitive to Ciprofloxacin. One month post-

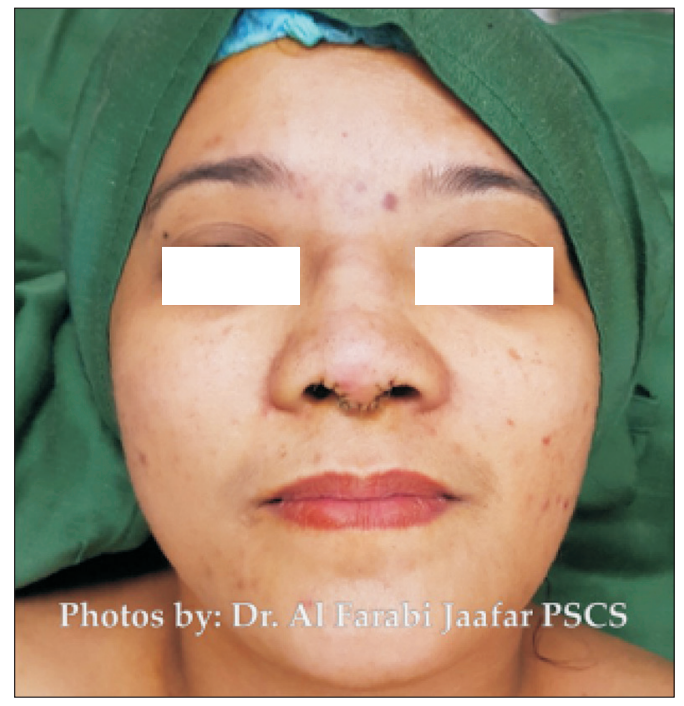

Fig. 3. Immediate post-surgery. 

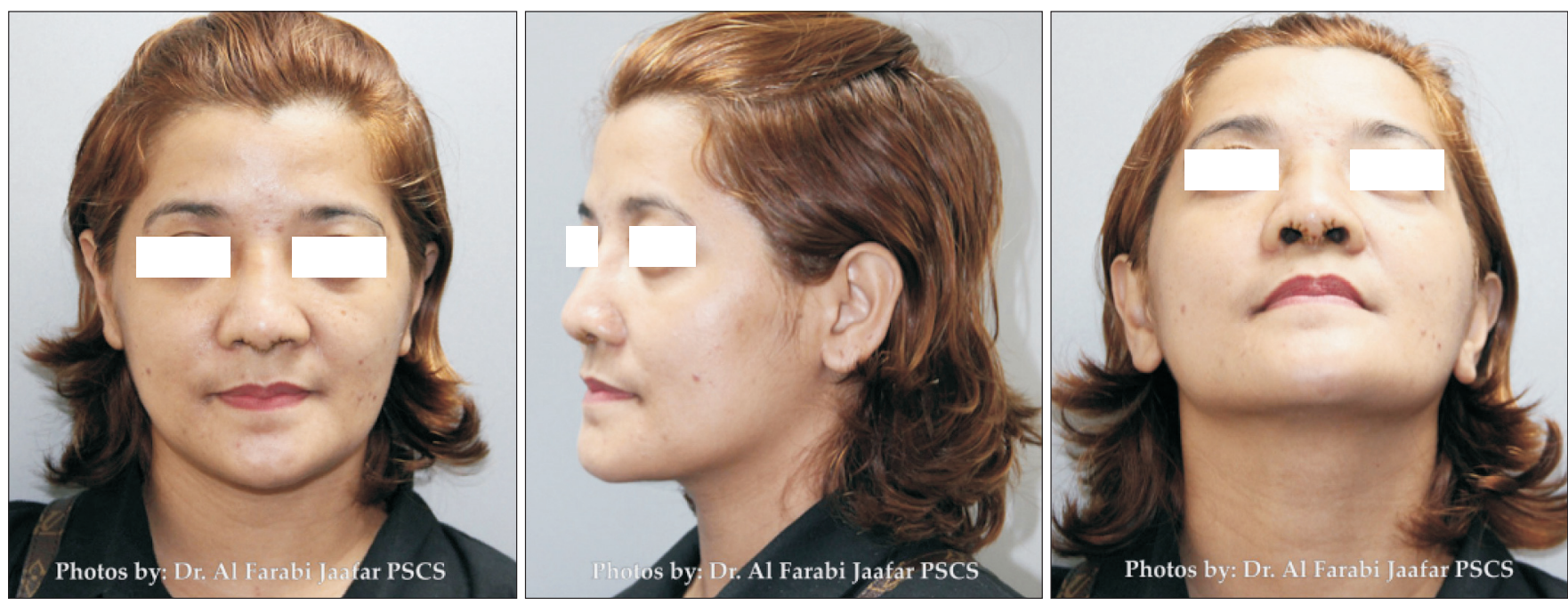

Fig. 4. Third day after rhinoplasty. Drainage of abscess.

surgery, the patient followed-up and had her photos taken (Fig. 4). No signs of inflammation or active infection were observed.

\section{Discussion}

Augmentation rhinoplasty is one of the most common cosmetic surgery procedures in Asia. International Society of Aesthetics Plastic Surgery statistics for 2016, shows rhinoplasty always placing among the top 5 most frequently performed procedure among East and Southeast Asian countries.

In an online article by the Dermatology Times in 2013, an increasing number of patients are asking for non-surgical alternative to rhinoplasty. This demand has led initially to the use of fillers injected into the nose for augmentation. Since fillers have serious complications like skin necrosis and blindness, many doctors are becoming skeptical with its use. Accidental intravascular injection of filler materials may cause an embolic event resulting to permanent blindness. This complication can occur when fillers are injected in the nose and surrounding areas. Doctors performing the treatment need to be knowledgeable of adjacent vessels in the injection site. As little as $0.01 \mathrm{ml}$ of material can fill a very small vessel of $0.05 \mathrm{~cm}$ diameter and reach the retinal artery to cause blindness [8].

The potential risks and pitfalls of fillers with its migratory property generated interest in the use of threads. Threads are used commonly in other parts of the face for rejuvenation. Since its invention in the 1990s, thread lifting has its roller coaster ride over the past many years. Early data on its use showed inconsistent outcomes and shortened effects. As newer devices (threads) were developed and better techniques introduced, outcome and longevity dramatically improved [9].

New generation threads (with or without cogs) have recently come out of the market. They are typically made of absorbable PDO, a common suture material. Other longer lasting materials are also available. Threads are better alternative to surgical augmentation rhinoplasty in select patients especially those preferring non-invasive augmentation. It is considerably a safer procedure compared to the use of fillers. Worldwide incidence of blindness after fillers injection is increasing and the most common area injected is the nose [8].

While there is ease in the technical use of threads, doctors oftentimes miss on the critical step of adequate skin preparation. The nose is one of the dirtiest and most colonized regions of the body. It is imperative to follow strict aseptic and antiseptic techniques during treatments to avoid inoculation. In one published report, microbes found in the nose may be a key indicator for future development of skin and soft-tissue infections even in remote areas of the body [10].

The skin being the largest organ in the body is colonized by a diverse habitat of microorganisms. The nose contains a high concentration of sebaceous glands that secrete sebum. The sebum supports the growth of facultative anaerobes such as $P$. acnes, a common skin commensal bacterium [11] present in our patient. Their existence deep in the sebaceous glands makes them somehow unreachable to ordinary prepping. According to Sebben [12], approximately twenty percent (20\%) of microbes on the skin are beyond the reach of surgical scrubs or antiseptics. This data concretize the fact that a small hole made through the skin during insertion of the cannula for thread procedures, provides access for these microorganisms to colonize 
the inner layers. A contaminated thread can cause life-threatening complications. Infections within the "triangle of death", an area bounded by the corners of the oral commissure and the glabella, can lead to cavernous sinus thrombosis.

The choice of different skin antiseptics is very crucial. Common skin antiseptics are either alcohol based (ethyl, propyl, or isopropyl alcohol), Iodine or Iodophor-based (Povidone-Iodine) or Chlorhexidines. Comparison of these antiseptics showed effectiveness for all, with alcohol-based antiseptics showing greater efficacy [13]. While it is true that microorganisms can adapt to a variety of environmental physical and chemical conditions, and can thus cause resistance, important issues including inadequate cleaning, incorrect product use, or ineffective infection control practices, cannot be underestimated. For instance, based on studies for Guideline for Disinfection and Sterilization in Healthcare Facilities (2008) by Rutala et al. [14], alcohol-based antiseptics need a minimum of 10 to 15 seconds exposure in order for their bactericidal or virucidal actions to take effect with concentration range of $30 \%$ to $100 \%$ [15].

With plenty of data on hand, surgeons may be able to erase doubts and fears in offering non-surgical options to patients seeking non-invasive rhinoplasty by knowing the limitations and complications of fillers. Likewise, being aware that threads are better alternatives, it is also sensible to be knowledgeable on the safe application of this device. One lesson learned from this case report is strict surgical antiseptic technique to avoid introduction of microbes into the access where the threads are inserted. To our understanding, there has not been a case reported yet regarding abscess formation after thread augmentation rhinoplasty.

\section{Conclusion}

Nose lifting or reshaping using PDO threads has shown promise as an alternative to nose filler injections. While fillers have their own specific indications for minor correction of defects like bumps or grooves, threads have better shaping and lifting capability coupled with extended longevity. It also does not have the propensity to migrate. As a less-invasive procedure, it becomes an indispensible backup tool for surgeons to their surgical (implant or non-implant) rhinoplasty. The ease of application, short downtime/recovery, and good outcome (comparable to surgical rhinoplasty), have given nose thread lifting a great potential for use on busy patients who are not willing to undergo invasive treatments. Its success though is not only dependent on the product or the insertion skills of the surgeon, but also on his knowledge and understanding of good skin aseptic and antiseptic techniques. Such foundation will minimize if not prevent the unwanted complication of posttreatment nose infection.

\section{Conflicts of interest}

The author has nothing to disclose.

\section{References}

1. Khoo BC. Augmentation rhinoplasty in the orientals. Plast Reconstr Surg 1964;34:81-8.

2. Soo G. Asian rhinoplasty 2 [Internet]. Hongkong: Rhinoplasty Archive, 2011 [cited 2017 Dec 4]. Available from: https://www. rhinoplastyarchive.com/articles/asian-rhinoplasty-2.

3. Lafaille P, Benedetto A. Fillers: contraindications, side effects and precautions. J Cutan Aesthet Surg 2010;3:16-9.

4. Carruthers JD, Fagien S, Rohrich RJ, Weinkle S, Carruthers A. Blindness caused by cosmetic filler injection: a review of cause and therapy. Plast Reconstr Surg 2014;134:1197-201.

5. Beleznay K, Carruthers JD, Humphrey S, Jones D. Avoiding and treating blindness from fillers: a review of the world literature. Dermatol Surg 2015;41:1097-117.

6. Chiam CT. The perils of non-surgical rhinoplasty: a safer approach needed? -a comparison between PDO nose threads and fillers [Internet]. Singapore: International Fellowship in Advanced Aesthetic Science (IFAAS), 2017 [cited 2017 Dec 4]. Available from: https://www.ifaas.co/single-post/2017/01/18/ The-Perils-of-Non-surgical-Rhinoplasty-A-Safer-ApproachNeeded--Review-of-Applying-PDO-Nose-Thread-LiftingCompares-to-Fillers.

7. Ali YH. Two years' outcome of thread lifting with absorbable barbed PDO threads: Innovative score for objective and subjective assessment. J Cosmet Laser Ther 2018;20:41-9.

8. Townshend A. Blindness after facial injection. J Clin Aesthet Dermatol 2016;9:E5-7.

9. Sarah T. Understanding thread lifting [Internet]. London: Aesthetics Journal, 2015 [cited 2017 Dec 7]. Available from: https:// aestheticsjournal.com/feature/understanding-thread-lifting.

10. Uniformed Services University of the Health Sciences (USU). Nasal bacteria may be predictor of skin infections [Internet]. Chicago: ScienceDaily, 2014 [cited 2018 Jan 7]. Available from: www.sciencedaily.com/releases/2014/05/140521094318.htm.

11. Grice EA, Segre JA. The skin microbiome. Nat Rev Microbiol 2011;9:244-53. 
12. Sebben JE. Surgical antiseptics. J Am Acad Dermatol 1983; 9:759-65.

13. Calfee DP, Farr BM. Comparison of four antiseptic preparations for skin in the prevention of contamination of percutaneously drawn blood cultures: a randomized trial. 2002;40:1660-5.
14. Rutala WA, Weber DJ, Healthcare Infection Control Practices Advisory Committee (HICPAC). Guideline for disinfection and sterilization in healthcare facilities. 2008.

15. McDonnell G, Russell AD. Antiseptics and disinfectants: activity, action, and resistance. Clin Microbiol Rev 1999;12:14779. 